\title{
Application of Adaptive Coded Modulation Technology in UAV Data Link
}

\author{
Rui Xue1, Deting Hu1, Tielin Zhu² \\ ${ }^{1}$ College of Information and Communication Engineering, Harbin Engineering University, Harbin, China \\ ${ }^{2}$ Tianjin Key Laboratory of Intelligent Information Processing in Remote Sensing, Tianjin, China \\ Email: zhutielin1234@163.com
}

How to cite this paper: Xue, R., Hu, D. and Zhu, T. (2017) Application of Adaptive Coded Modulation Technology in UAV Data Link. Int. J. Communications, Network and System Sciences, 10, 181-190. https://doi.org/10.4236/ijcns.2017.105B018

Received: April 10, 2017

Accepted: May 5, 23

Published: May 5, 26

\begin{abstract}
UAV data link has been considered as an important part of UAV communication system, through which the UAV could communicate with warships. However, constant coding and modulation scheme that UAV adopts does not make full use of the channel capacity when UAV communicates with warships in a good channel environment. In order to improve channel capacity and spectral efficiency, adaptive coded modulation technology is studied. Based on maritime channel model, SNR estimation technology and adaptive threshold determination technology, the simulation of UAV data link communication is carried out in this paper. Theoretic analysis and simulation results show that according to changes in maritime channel state, UAV can dynamically adjust the adaptive coded modulation scheme on the condition of meeting target Bit-Error-Rate (BER), the maximum amount of data transfer is non-adaptive systems three times.
\end{abstract}

\section{Keywords}

UAV Data Link, Adaptive Coded Modulation, Maritime Channel, SNR Estimation, Target Bit-Error-Rate

\section{Introduction}

Compared with manned aircraft, unmanned aerial vehicles have advantages of strong endurance, affordable, no casualties and low-maintenance and training cost. With the progress of UAV technology, more and more UAVs are used for military reconnaissance, military combat and other tasks.

UAV data link has been considered as an important part of UAV communication system, its main task is to establish a bidirectional data transmission link between base station and UAV, and complete information transmission, which include the UAV remote control information, telemetry and reconnaissance in- 
formation. Therefore, the reliability and efficient of UAV data link is one of the key factors that determine the performance of the UAV.

January 2013, the US Air Force Life Cycle Management Centre announced the new common data link (CDL) project, which aimed to seek future program of common data link, including determining the performance of parameters to improve the future combat capacity of the Air Force. According to the requirements of US Air Force, future common data link's capacity should have the following three aspects: multi-access network, adaptive parameters and the capability of communication support. Adaptive parameters consist of a wide variety of flight control parameters, such as data rate, transmission power, modulation scheme and error correction coding, frequency and other parameters, if RF environment changes, parameters should adaptively change to adapt to the new channel environment.

The communication channel between UAV and base station is a complex dynamic channel, in order to ensure the normal communication, the style of channel coding and modulation are selected with the worst channel condition and the lowest channel standard [1]. In this case, channel capacity can't be effectively used when the channel environment changes better. In order to improve channel capacity and spectral efficiency, adaptive coding and modulation techniques are studied [2].

The rest of paper is organized as follows. In Section 2, the model of maritime channel and wave simulation are described. Section 3 introduces a method of estimating SNR. Adaptive coding and modulation scheme and its performance simulation curves are discussed in Section 4. Finally, we conclude the paper in Section 5 .

\section{Maritime Channel Simulation}

The communication channel between UAV and warships is a time-varying, complex wireless mobile communication channel, fluctuating waves will change the direction of propagation of the signal, and part of the signal through the reflection of the waves can still reach the receiver. Therefore, the receiver will receive signals of different paths, resulting in multipath fading [3]. To determine the effect of maritime channel on communication system, this section will focus on the multi-path model and wave simulation, on this basis, the size of the maritime channel loss is given.

\subsection{Multipath Model}

Due to the impact of waves, received signals of sea receiver contain not only the direct signal, but also comprise signals from the vast sea through different points of reflection over the different paths. Signals of different paths arrive at the receiver in different time, and they will also have different carrier phase and different power. Since the signal that receiver finally receive is the vector sum of the respective signal paths, a multipath channel model is used to describe the maritime communication channel in this paper [4] [5]. 
Assume that the signal received by the receiver is a vector sum of $\mathrm{N}$ signal paths, so signal receiving power could be expressed as

$$
P_{r}=P_{t}\left(\frac{\lambda}{4 \pi d}\right)^{2} g_{r} g_{t}\left|\sum_{i=1}^{N} \operatorname{Re}^{j \Delta \phi_{i}}\right|^{2}
$$

where $P_{r}$ is the received power, $P_{t}$ is transmitted power, $g_{r}$ is the gain of transmitting antenna, $g_{t}$ is the gain of receiving antenna, $\Delta \phi$ is the difference of phase.

Phase difference could be calculated by Equation (2):

$$
\Delta \phi=\frac{2 \pi \Delta l}{\lambda}
$$

$\Delta l$ is different between the reflected path and the direct path, its calculation method will be introduced in the following chapter.

\subsection{Wave Simulation}

The communication is inevitably affected by storms at sea when UAV communicate with warships, in order to ensure the smooth conduct of maritime communications, we must study the impact of the waves on the communication. As the premise of accurate study of the influence of ocean wave on the communication is to establish the wave model which can reflect the real wave. We use Pierson Moskowitz spectrum the standard wave spectrum that ITTC prescribe as a target spectrum to study and simulate three-dimensional random wave.

Movement of the waves is a complex three-dimensional stochastic process; the wave is regarded as the cosine wave superposition of many different wave heights, different periods, different phases, and different direction for the sake of simulating the three-dimensional random wave accurately. We use $z=\varphi(x, y, t)$ to describe the equation of wave surface, and then the wave surface express can be expressed as follows:

$$
\varphi(x, y, t)=\sum_{i=1}^{M} \sum_{j=1}^{N} \xi_{i j}\left[\cos \left(x \cos \alpha_{j}+y \sin \alpha_{j}\right)-\omega_{i} t+\beta_{i j}\right]
$$

where $\xi_{i j}$ is the amplitude of waves, $k_{i}$ are numbers of waves, $\alpha_{j}$ is the direction angle, $\omega_{i}$ is the frequency, $\beta_{i j}$ is the phase angle. The determination of these parameters in the references [6] [7] is described in detail.

The appropriate wave spectrum as the target spectrum should be first selected before the simulation of waves, in this paper we use (4) to describe target spectrum.

$$
S(\omega, \alpha)=S_{P M}(\omega) \cdot D_{f}(\alpha)
$$

where $S_{P M}(\omega)$ is the Pierson-Moskowitz spectrum the standard wave spectrum that ITTC prescribe, which describes the energy changes with frequency, $D_{f}(\alpha)$ is extended spectrum function for wave spectrum.

$$
\begin{array}{r}
\mathrm{S}_{P M}(\omega)=\frac{0.78}{\omega^{5}} \exp \left[-\frac{3.11}{\omega^{4} H_{1 / 3}^{2}}\right] \\
D_{f}(\alpha)=\frac{2}{\pi} \cos ^{2} \alpha,\left(-\frac{\pi}{2} \leq \alpha \leq \frac{\pi}{2}\right)
\end{array}
$$


Combining Equation (3), (4), (5) and Equation (6), we can simulate different levels of ocean waves by using MATLAB software. The randomly generated sea surface for the sea state level of three is shown in Figure 1(a), and the sea state level of five is shown in Figure 1(b).

\subsection{The Calculation of Key Parameters and Channel Loss}

In this paper, the multipath model is used as the model of the maritime mobile channel, and the key to realize the multipath model is to calculate the reflection coefficient and phase different. As the premise of calculating the reflection coefficient and phase difference is to determine the position of the reflection point, Newton iterative method is used to calculate the position of reflection point in this paper.

Newton iterative method is an important and commonly used iterative method, its basic idea is gradually linearizing nonlinear function $f(x)$, so as to

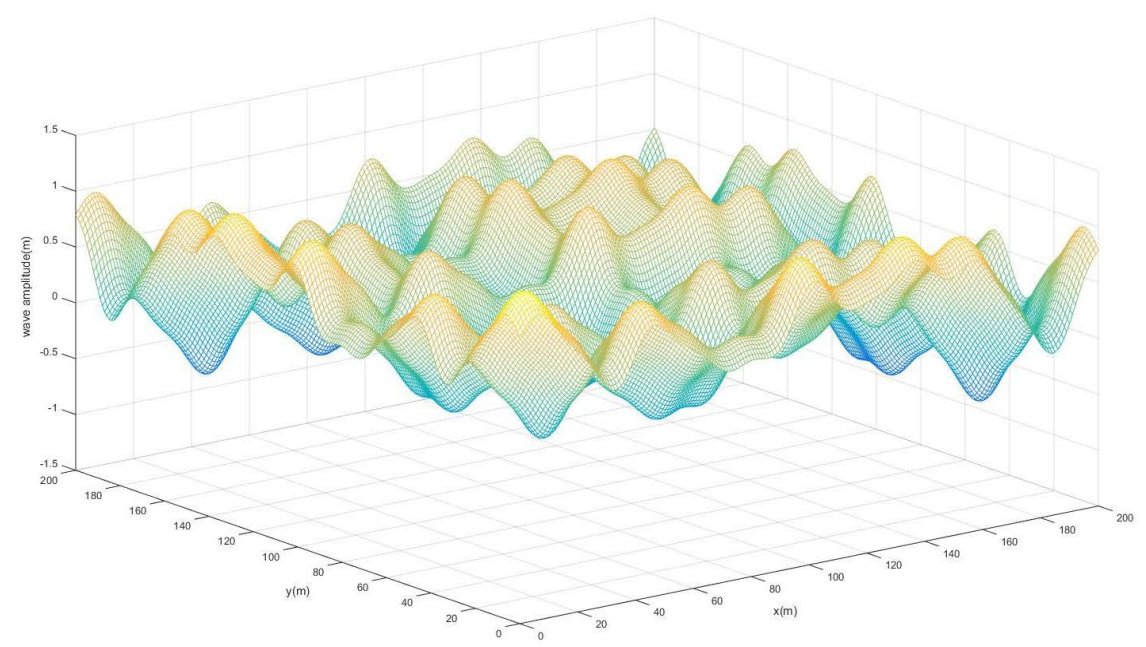

(a)

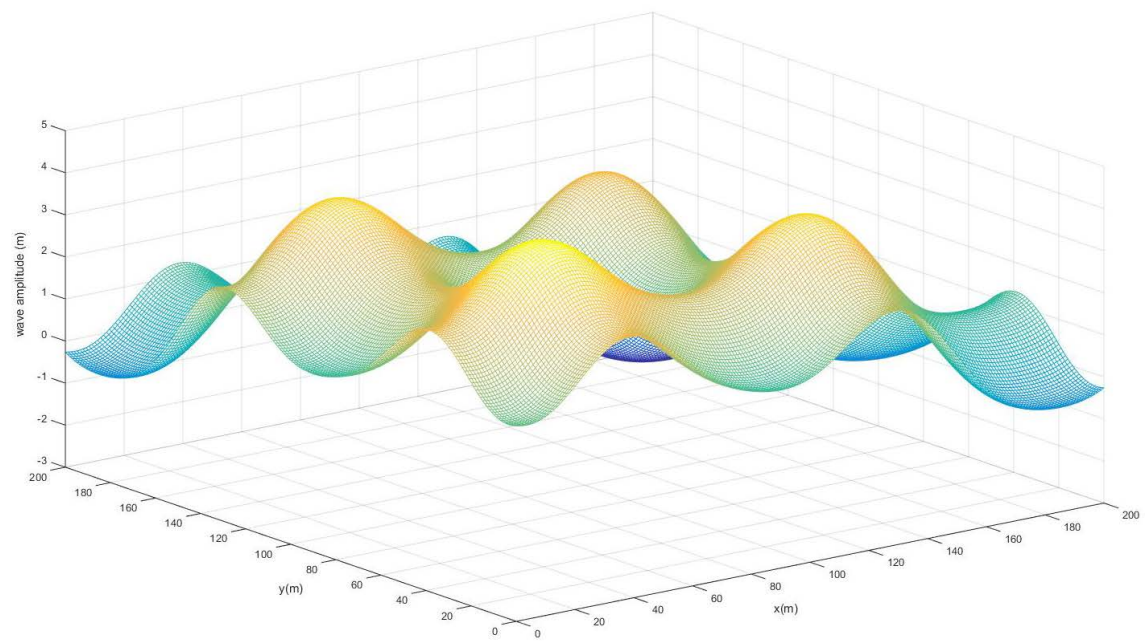

(b)

Figure 1. The randomly generated sea surface. (a) The sea surface of sea level three; (b) The sea surface of sea level five. 
transform the nonlinear equation $f(x)=0$ approximate into liner equation to solve. The process to solve is as follows.

Assume that $x_{k}$ is the approximate root of $f(x)=0$, so the function $f(x)$ can be done Taylor expansion in the vicinity of $x_{k}$ :

$$
f(x)=f\left(x_{k}\right)+f^{\prime}\left(x_{k}\right)\left(x-x_{k}\right)+\frac{1}{2} f^{\prime \prime}\left(x_{k}\right)\left(x-x_{k}\right)^{2}+\cdots
$$

Ignoring the higher-order terms, use the liner part as the approximate representation of function $f(x)$ :

$$
f(x)=f\left(x_{k}\right)+f^{\prime}\left(x_{k}\right)\left(x-x_{k}\right)
$$

Hypothesis that $x^{*}$ is the root of function $f(x)=0$, so $f\left(x^{*}\right)=0$, that is to say $f\left(x_{k}\right)+f^{\prime}\left(x_{k}\right)\left(x^{*}-x_{k}\right) \approx 0$, so we can get the approximate expression of $x^{*}:$

$$
x^{*} \approx x_{k}-\frac{f\left(x_{k}\right)}{f^{\prime}\left(x_{k}\right)}
$$

So we can get the famous Newton iterative formula:

$$
x_{k+1} \approx x_{k}-\frac{f\left(x_{k}\right)}{f^{\prime}\left(x_{k}\right)}
$$

Equation (10) will not be stopped until the root new produced meet the requirements of $\left|f\left(x_{k}\right)\right| \leq \varepsilon$.

Newton iterative method can be used to get the intersection point of the incident wave and the ocean wave. Assuming the elevation angle of the incident wave is $\theta, b$ is intercept, so the incident wave can be presented as Equation (12):

$$
f_{1}(x)=\tan \theta * x+b
$$

So, the expression of $f(x)$ is:

$$
f(x)=f_{1}(x)-f_{2}(x)
$$

where $f_{2}(x)$ is the wave expression, as shown in Equation (3).

During the research, $b$ will increase in the small increments gradually and to find the point of intersection through continuous iteration. As how to judge that whether the intersection point is the reflection of the point is not the focus of this paper, therefore, this section will no longer be described in detail.

After finding the position of the reflection point, the path difference between the reflected wave and the direct wave can be obtained by the simple calculation according to the geometric relationship:

$$
\Delta l=\frac{h}{\beta} *[1-\cos (\theta+\beta)]
$$

where $\beta$ is the elevation angle of the reflected wave.

Combining Equations (1), (2) and Equation (13), channel loss can be derived from Equation (14):

$$
L=10 \log \left(\frac{P_{t}}{P_{r}}\right)=-10 \log \left[\left(\frac{\lambda}{4 \pi d}\right) g_{r} g_{t}\left|1+\sum_{i=1}^{N} \operatorname{Re}^{j \Delta \phi_{i}}\right|^{2}\right]
$$




\section{Channel Estimation}

Channel estimation is to estimate the actual channel state information by using the indeed known information of the signal [8]. Since the adaptive coding and modulation scheme switch its scheme adaptively based on the estimated channel information under different channel conditions, therefore, the channel estimation algorithm has a direct impact on the effectiveness of the adaptive coding and modulation method. In this paper, we use the data fitting SNR estimation method based on statistics to estimate the channel state [9].

By establishing the relationship of the second moment between the real part of the received signal and the signal to noise ratio, we can get the relationship of the SNR estimation through the use of the data fitting SNR estimation method. The expression of the received signal is: $y=f x+n$, where, $n$ represents noise, $f$ is the fading factor. When the channel is AWGN channel, $f$ is constant, at this point for the MPSK signal, the received signal according to the real and imaginary parts can be expressed respectively as follows:

$$
r_{k_{-} I / Q}= \pm A+n_{k}, \quad k=1,2, \cdots, L
$$

where, $I, Q$ represents the real and imaginary part of the signal respectively, the probability of taking the positive and negative in Equation (15) are equal, both for $1 / 2$. The signal to noise ratio to be estimated is defined as:

$$
S N R=\frac{\left|a_{k}\right|^{2}}{2 \sigma^{2}}=\lambda
$$

Owning to

$$
\begin{gathered}
\lambda=\frac{\left|a_{k}\right|^{2}}{2 \sigma^{2}}=\frac{\operatorname{real}\left(a_{k}\right)^{2}+\operatorname{imag}\left(a_{k}\right)^{2}}{2 \sigma^{2}} \\
=\frac{A^{2}}{\sigma^{2}}=S N R_{I / Q}
\end{gathered}
$$

We can derive $S N R=S N R_{I / Q}$ from Equation (15) and (16), that is to say the SNR of the complex signal is the same as the SNR of the real (imaginary) part of the signal. Therefore, when calculating the signal to noise ratio at the receiving end, we just need to estimate the SNR to the real or imaginary part.

The expression of $z$ is as follows:

$$
\begin{gathered}
Z=E\left(r_{k_{-} I / Q}^{2}\right) /\left[E\left(\left|r_{k_{-} I / Q}\right|\right)\right]^{2} \\
E\left(r_{k_{-} I / Q}^{2}\right)=A^{2}+\sigma^{2} \\
E\left(\left|r_{k_{-} I / Q}\right|\right)=\sigma \sqrt{\frac{2}{\pi}} e^{-\left(\frac{A^{2}}{2 \sigma^{2}}\right)}+A\left[\operatorname{erf} \sqrt{\frac{A^{2}}{2 \sigma^{2}}}\right]
\end{gathered}
$$

From Equations (17), (18), (19) and (20), we can derive Equation (21):

$$
z=\frac{E\left(r_{k_{-} I / Q}^{2}\right)}{E\left[\left|r_{k_{-} I / Q}\right|\right]^{2}}=\frac{1+\lambda}{\left\{\sqrt{\frac{2}{\pi}} e^{-\frac{\lambda}{2}}+\sqrt{\lambda}\left[\operatorname{erf}\left(\sqrt{\frac{\lambda}{2}}\right)\right]\right\}^{2}}=f(\lambda)
$$


We use $z$ to calculate $\lambda(\lambda=g(z))$, because of the complexity of the Equation (21), the expression of $\lambda=g(z)$ can't be directly obtained. Therefore we can get the polynomial approximate expression over a period ranging of $\lambda$ by using the data fitting SNR estimation method. Through comparative analysis, we choose Equation (22) $5^{\text {th }}$ order polynomial to approximate $g(z)$, its accuracy is shown in Figure 2. Compared with the actual SNR, the signal to noise ratio obtained by the data fitting estimation method is shown in Figure 3.

$$
\begin{gathered}
\lambda=10^{4}\left(-0.41292 \times z^{5}+2.66418 \times z^{4}-6.86724 \times z^{3}+\right. \\
\left.8.84039 \times z^{2}-5.68658 \times z+1.46404\right)
\end{gathered}
$$

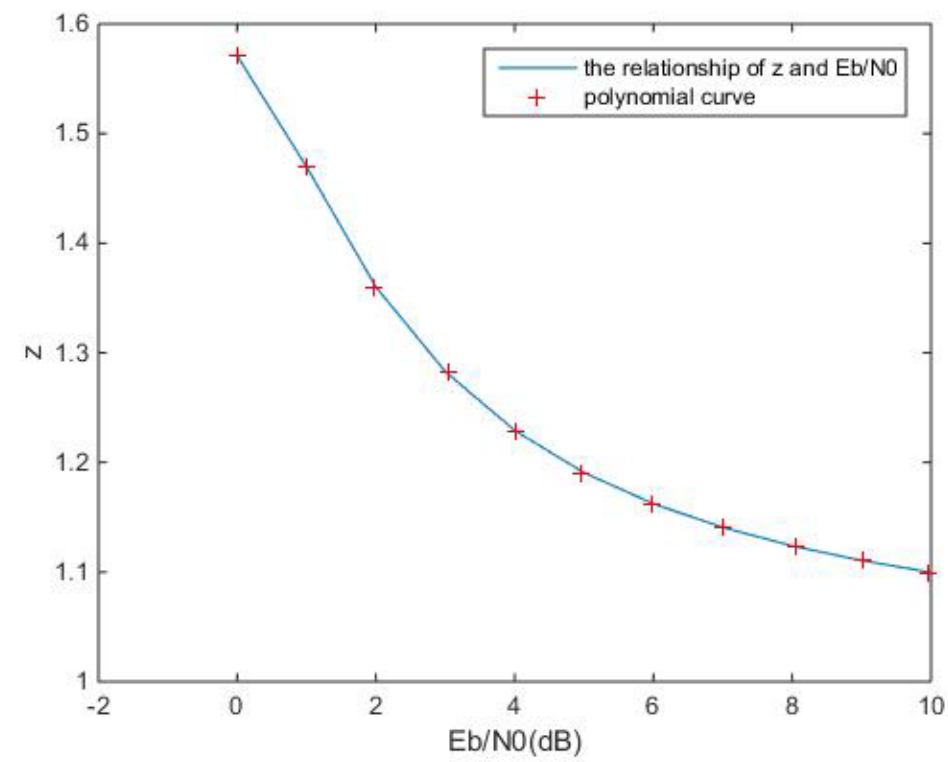

Figure 2. The accuracy of polynomial curve.

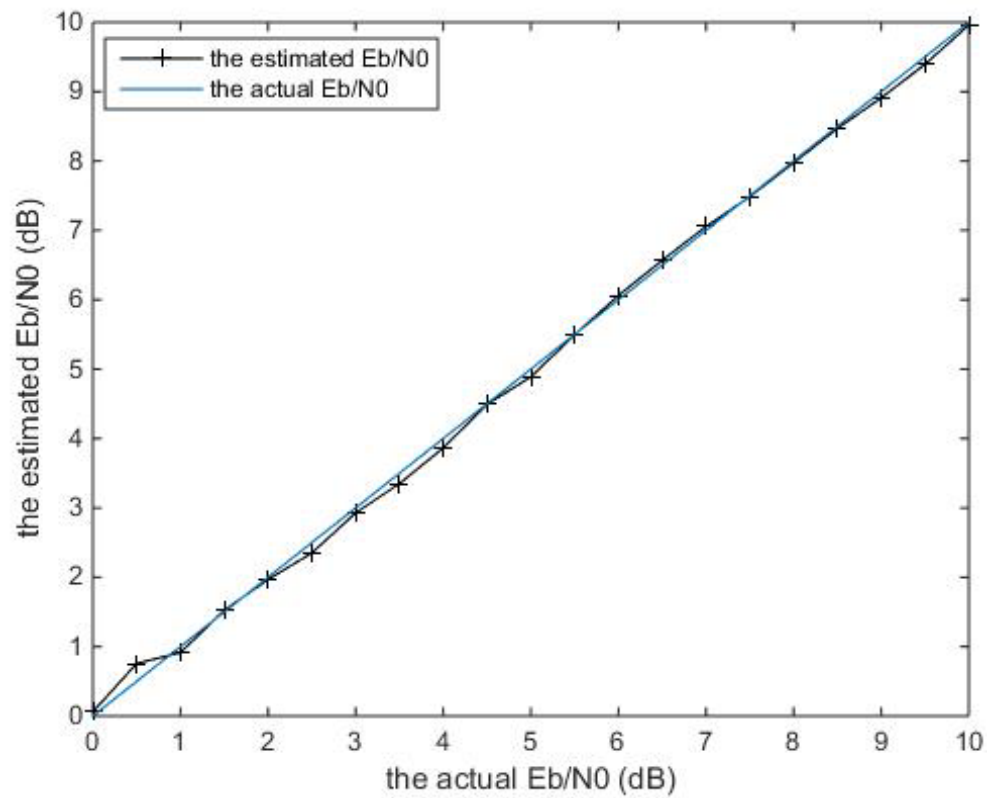

Figure 3. The comparison of actual Eb/N0 and estimated Eb/N0. 
For Equation (22), $Z$ can use an average of the received data to replace the mean, that is $z \approx \overline{r_{k_{-} I / Q}^{2}} / \mid \overline{\left.r_{k_{-} I / Q}\right|^{2}}$, as $\overline{r_{k_{-} I}^{2}} /\left|\overline{\left.r_{k_{-} I}\right|^{2}} \approx \overline{r_{k_{-} Q}^{2}} /\right| \overline{\left.r_{k_{-} Q}\right|^{2}}$ for the modulation scheme of MPSK, so, in order to make full use of all the useful information, the approximate value of $Z$ is expressed as follows:

$$
\mathrm{Z} \approx \frac{\overline{r_{k_{-} I}^{2}}}{{\overline{\mid r_{k_{-} I}}}^{2}} \approx \frac{\overline{r_{k_{-} Q}^{2}}}{\overline{\left|r_{k_{-} Q}\right|^{2}}} \approx \frac{\overline{r_{k_{-} I}^{2}}+\overline{r_{k_{-} Q}^{2}}}{\left|\overline{r_{k_{-} I} \mid}\right|^{2}+\overline{\left|r_{k_{-} Q}\right|^{2}}}
$$

\section{Adaptive Coded Modulation Scheme and Performance Simulation}

The basic idea of adaptive coded modulation technique is to estimate the channel state at the receiving end, then the estimated information of channel state will be transmitted back to the transmitter, last, channel coding mode and modulation mode are changed according to the channel state information at the transmitter [10] [11]. Therefore, the adaptive coded modulation system can transmit data at high speed in favorable channel, and reduce the throughout when the channel becomes poor, thus it can provide a high average channel spectrum efficiency at the premise of not sacrificing the system power and bit error rate.

Currently encoding Navy UAV data link used and being developed mainly employ convolution code, modulation mode mainly is OQPSK. Therefore, this paper will also simulate the performance of the convolution code and OQPSK. In addition, the LDPC code with high gain coding performance is also used in this paper, the rate is $1 / 2,1 / 3,1 / 4,2 / 3,3 / 4,2 / 5,3 / 5,4 / 5$, respectively. In order to improve the effectiveness of the system in the favorable channel, the performance of LDPC code and the convolutional coding in the 8PSK are simulated, too.

When the error rate is $10^{-5}$, the fixed threshold of the adaptive coded modulation can be determined according to Figure 4, as shown in Table 1.It can be seen from Table 1 that the MCS2 scheme is adopted when the signal to noise is higher than $3.4 \mathrm{~dB}$. In this case the information of each symbol transmitted by transmitter is $2 / 3$ bit; the MCS4 scheme is adopted when the signal to noise ratio is higher than $5.1 \mathrm{~dB}$, the information of each symbol is $6 / 5$ bits; the MCS5 scheme is adopted when the signal to noise ratio is higher than $6.4 \mathrm{~dB}$, the information of each symbol is $9 / 4$ bits; when the signal to ratio is higher than $7.6 \mathrm{~dB}$, the MCS6 scheme is adopted, the information is $12 / 5$ bits. When the channel state is good, the scheme of large through is adopted to improve the system throughput. When the channel is poor, the low throughput scheme is adopted to ensure the BER performance of the system. With the channel state changing adaptively change the modulation and coding scheme, so that the system can get higher throughput than adopting the fixed coding modulation scheme of convolutional coding and OQPSK. As the maximal $R_{n}$ of adaptive coded modulation is $12 / 5$ bits and the $R_{n}$ of the fixed coding modulation is $2 / 3$, therefore, the amount of data transmitted by the adaptive coded modulation technology can be 3.6 times as great as that of the non-adaptive system. 


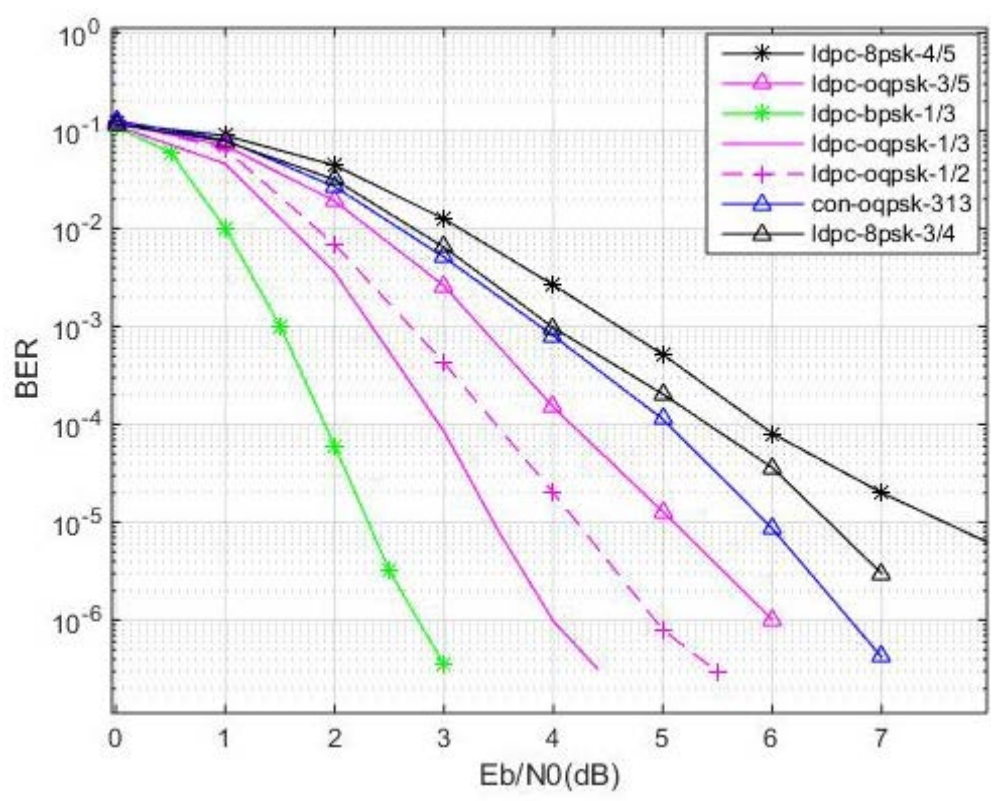

Figure 4. BER curves under different modulation schemes.

Table 1. The scheme of adaptive coded modulation.

\begin{tabular}{ccccc}
\hline SNR & Modulation & rate & $R_{n}$ & Scheme \\
\hline $2.2-3.4$ & BPSK & $1 / 3$ & $1 / 3$ & MCS1 \\
$3.4-4.3$ & & $1 / 3$ & $2 / 3$ & MCS2 \\
$4.3-5.1$ & OQPSK & $1 / 2$ & 1 & MCS3 \\
$5.1-6.4$ & & $3 / 5$ & $6 / 5$ & MCS4 \\
$6.4-7.6$ & & $3 / 4$ & $9 / 4$ & MCS5 \\
$7.6-$ & 8PSK & $4 / 5$ & $12 / 5$ & MCS6 \\
\hline
\end{tabular}

\section{Conclusion}

In order to improve the system throughput when the channel is in good condition, this paper studies the adaptive coding and modulation technology based on LDPC code. In order to realize the dynamic selection of coded modulation scheme, the paper also studies the technology of the maritime channel model and the SNR estimation. The adaptive scheme is adaptively selected based on the channel state that estimated through the SNR estimation technique, so that the system throughput can be improved effectively under the premise of guaranteeing the bit error rate. Through the simulation and theoretical analysis, we conclude that the amount of data transmitted by the adaptive coded modulation technology can be 3.6 times as great as that of the non-adaptive system.

\section{Acknowledgements}

This paper is funded by the International Exchange Program of Harbin Engineering University for Innovation-oriented Talents Cultivation, the Open Research Fund of State Key Laboratory of Tianjin Key Laboratory of Intelligent 
Information Processing in Remote Sensing (Grant No. 2016-ZW-KFJJ-01), the National Natural Science Foundation of China (Grant No. 61403093), the Assisted Project by Heilongjiang Province of China Postdoctoral Funds for Scientific Research Initiation (Grant No. LBH-Q14048), and the Fundamental Research Funds for the Central Universities (Grant No. HEUCF160813).

\section{References}

[1] Bobbie, P.O. (2014) Adaptive Modulation and Coding for Efficient Wireless Channel Capacity Utilization. International Conference on Computer Applications in Industry and Engineering, CAINE, 199-205.

[2] Kim, J. and Cho, D.H. (2005) Enhanced Adaptive Modulation and Coding Schemes Based on Multiple Channel Reportings for Wireless Multicast Systems. IEEE Vehicular Technology Conference, Vtc-2005-Fall, 725-729.

[3] Yu, G.F. (2014) Study on Wireless Channel Models of UAV Data Link. Applied Mechanics \& Materials, 543-547, 2605-2608. https://doi.org/10.4028/www.scientific.net/AMM.543-547.2605

[4] Cao, X.D. and Jiang, T. (2014) Research on Sea Surface Ka-Band Stochastic Multipath Channel Modeling. IEEE Antennas and Propagation, 675-678. https://doi.org/10.1109/apcap.2014.6992586

[5] Huang, F., Liao, X. and Bai, Y. (2016) Multipath Channel Model for Radio Propagation over Sea Surface. Wireless Personal Communications, 1-13. https://doi.org/10.1109/TWC.2016.2547861

[6] He, H.Q. (2005) A Way to Real-Time Ocean Wave Simulation. IEEE International Conference on Computer Graphics, Imaging and Vision: New Trends, 409-415.

[7] Hu, Z., Tang, W. and Xue, H. (2014) A Probability-based Superposition Model of Freak Wave Simulation. Applied Ocean Research, 47, 284-290. https://doi.org/10.1016/j.apor.2014.05.007

[8] Chen, F.J. (2014) Non-Data-Aided ML SNR Estimation for AWGN Channels with Deterministic Interference. Eurasip Journal on Wireless Communications \& Networking, 2014, 1-10. https://doi.org/10.1186/1687-1499-2014-45

[9] Ramesh, A., Chockaligam, A. and Milstein, L.B. (2001) SNR Estimation in Generalized Fading Channels and Its Application to Turbo Decoding.

[10] Gregory, M. and Hoeher, P.A. (2011) Adaptive Channel Coding for Maritime FSO Channels with RF Feedback Link. International Conference on Space Optical Systems and Applications, 351-357. https://doi.org/10.1109/icsos.2011.5783696

[11] Rico-Alvarino, A., Tato, A. and Mosquera, C. (2015) Robust Adaptive Coding and Modulation Scheme for the Mobile Satellite Forward Link. IEEE International Workshop on Signal Processing Advances in Wireless Communications. https://doi.org/10.1109/spawc.2015.7227094 
Submit or recommend next manuscript to SCIRP and we will provide best service for you:

Accepting pre-submission inquiries through Email, Facebook, LinkedIn, Twitter, etc. A wide selection of journals (inclusive of 9 subjects, more than 200 journals)

Providing 24-hour high-quality service

User-friendly online submission system

Fair and swift peer-review system

Efficient typesetting and proofreading procedure

Display of the result of downloads and visits, as well as the number of cited articles Maximum dissemination of your research work

Submit your manuscript at: http://papersubmission.scirp.org/

Or contact ijens@scirp.org 